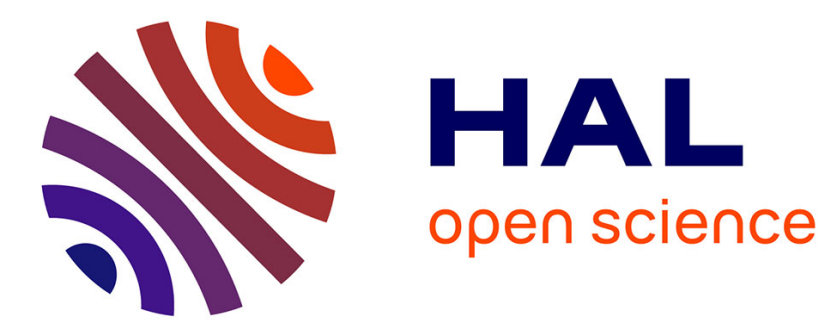

\title{
Un tympan roman à l'île-Barbe près de Lyon
}

Robert Favreau

\section{To cite this version:}

Robert Favreau. Un tympan roman à l'Île-Barbe près de Lyon. Comptes-rendus des séances de l'Académie des inscriptions et belles-lettres, 2005, 149 (3), pp.1007-1025. 10.3406/crai.2005.22911. halshs-03199206

\section{HAL Id: halshs-03199206 https://shs.hal.science/halshs-03199206}

Submitted on 15 Apr 2021

HAL is a multi-disciplinary open access archive for the deposit and dissemination of scientific research documents, whether they are published or not. The documents may come from teaching and research institutions in France or abroad, or from public or private research centers.
L'archive ouverte pluridisciplinaire HAL, est destinée au dépôt et à la diffusion de documents scientifiques de niveau recherche, publiés ou non, émanant des établissements d'enseignement et de recherche français ou étrangers, des laboratoires publics ou privés. 


\section{Un tympan roman à l'île-Barbe près de Lyon}

\section{Robert Favreau}

\section{Citer ce document / Cite this document :}

Favreau Robert. Un tympan roman à l'Île-Barbe près de Lyon. In: Comptes rendus des séances de l'Académie des Inscriptions et Belles-Lettres, 149 ${ }^{\mathrm{e}}$ année, N. 3, 2005. pp. 1007-1025;

doi : https://doi.org/10.3406/crai.2005.22911

https://www.persee.fr/doc/crai_0065-0536_2005_num_149_3_22911

Fichier pdf généré le 22/05/2018 


\title{
COMMUNICATION
}

\author{
UN TYMPAN ROMAN À LîLE-BARBE PRÈS DE LYON, \\ PAR M. ROBERT FAVREAU, CORRESPONDANT DE L'ACADÉMIE
}

L'île-Barbe, insula barbara, est une île au milieu de la Saône, peu avant son confluent avec le Rhône. Elle mesure $560 \mathrm{~m}$ en longueur, 125 en largeur. Un monastère Saint-André y a été établi au temps de saint Eucher, évêque de Lyon, dans la première moitié $d u v^{e}$ siècle. Il adopta la règle de Saint-Benoît à la fin du $\mathrm{VII}^{\mathrm{e}}$ siècle. Restauré sous Charlemagne, il apparaît, à partir $\mathrm{du}^{\mathrm{e}}$ siècle, placé sous le patronage de saint Martin. L'abbaye a été sécularisée en $1549^{1}$. Aujourd'hui l'emplacement de l'abbaye et les restes des bâtiments abbatiaux sont occupés par des propriétaires privés, en un ensemble fermé chaque soir. Le site est classé.

Le précis historique donné par l'abbé Roux dans le Bulletin monumental en 1844 fait état d'« une porte romane d'une belle exécution qui était l'ancienne porte du réfectoire ", placée dans le mur d'appui du cloître, et il donne les inscriptions qui figuraient autour du tympan. Pour compliquer le problème de localisation, Victor Terret écrit en 1914 que le tympan roman de l'île-Barbe est placé aujourd'hui à la façade méridionale de l'église Saint-Rambert-l' ̂̂le-Barbe, et Philippe Verdier, en 1982, donne ce tympan roman comme celui de l'église abbatiale ${ }^{2}$. Lorsque nous sommes allés étudier sur place les inscriptions du département du Rhône, en mai 1987, nous avons parcouru la grande rue qui traverse l'île, nous avons, en vain, essayé de joindre les propriétaires de l'église Notre-Dame située sur cette grande rue, et, finalement nous avons dû publier les textes des

1. Abbé Roux, «Précis historique sur l'Île-Barbe ", Bulletin monumental, t. 10 (1844), p. $65-84$; L. Niepce, L'Île-Barbe, son ancienne abbaye et le bourg de Saint-Rambert. Lyon, 1890.

2. V. Terret, La sculpture bourguignonne aux XF et XIr siècles. Ses origines et ses sources d'inspiration. Cluny-Autun-Paris, 1914, p. 27 et n. 7 ; Ph. Verdier, "Dominus potens in proelio ", Wallraf-Richartz-Jahrbuch 43 (1982), p. 51. 
inscriptions de cette porte romane à partir du relevé de l'abbé Roux $^{3}$. Et puis il y a quelques années un de mes fils s'est installé à Lyon, et un dimanche nous avons emmené les petits-enfants à l'Île-Barbe, pour qu'ils puissent profiter de la tranquille aire de jeux installée dans l'espace vert qui occupe le tiers de l'île. Après avoir flâné dans la grande rue qui forme l'axe de l'ensemble habité nous sommes allés voir les bâtiments anciens du côté nord et nous avons emprunté l'impasse Saint-Louis, parallèle à la Saône. Et nous avons trouvé là, parfaitement accessible à tous les visiteurs, le mur du cloître de l'église principale disparue SaintMartin-et-Saint-Loup, avec l'ancienne porte romane du réfectoire. A la porte de la façade méridionale de l'église de Saint-Rambert un fragment de tympan roman, Christ au nimbe crucifère dans une mandorle, a été réemployé dans un tympan du $\mathrm{XIX}^{\mathrm{e}}$ siècle. Sans doute s'agit-il d'un fragment provenant de l'îleBarbe, d'où la confusion faite par Victor Terret.

Le plan de 1742, conservé aux Archives départementales du Rhône ${ }^{4}$, la reconstitution de l'ingénieur typographe J.-J. Grisard en 1889, la description de l'abbé Roux en 1844, de Léopold Niepce en 1890, permettent de situer la porte romane, objet de cette étude, et son contexte. Le réfectoire se trouvait du côté nord de l'île, près du port. Le bas de ses murs portés par cinq arcades ogivales était baigné par les eaux du petit bras de la Saône. Rebâti en 1507, il fut ruiné en 1562. Il jouxtait la partie occidentale de l'église abbatiale Saint-Martin, sur le flanc sud de laquelle avait été construit le grand cloître. En 1844 une allée de mûriers - l'impasse actuelle - avait remplacé les voûtes du cloître. Le mur qui longeait cette promenade était le mur d'appui du grand cloître. Ce fut dans ce mur que l'on installera, après la Révolution, la porte romane du réfectoire.

Le mur sud du grand cloître (fig. 1) est orné de grandes arcades, l'une en plein cintre reposant sur des pilastres cannelés, trois en arc brisé avec minces colonnettes à chapiteaux de feuillage. A gauche de la porte romane est placé un bas-relief mutilé où étaient représentés Bacchus, Pan et le dieu Sylvain. A droite de la porte on peut lire l'épitaphe d'un vétéran de la

3. R. Favreau. J. Michaud et B. Mora (éd.), Corpus des inscriptions de la France médiévale 17, Paris, 1994, p. 95.

4. Le plan est publié dans la Grande pancarte ou cartulaire de l'fle-Barbe.... éd. par le comte de Charpin-Feugerolles et G. Guigue, t. I, Montbrison, 1923. 


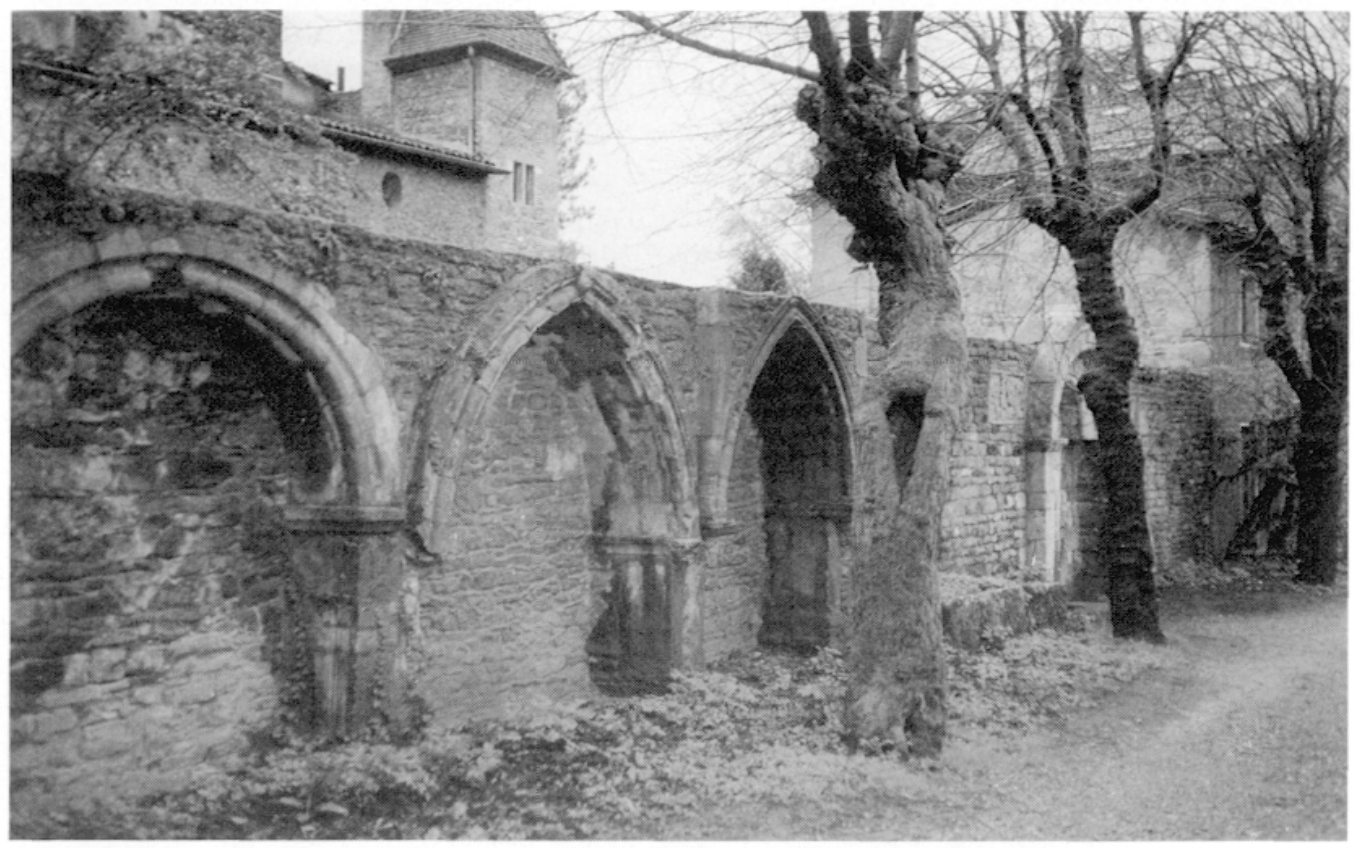

FIG. 1. - Le mur du cloître et la porte romane. Cl. R. Favreau.

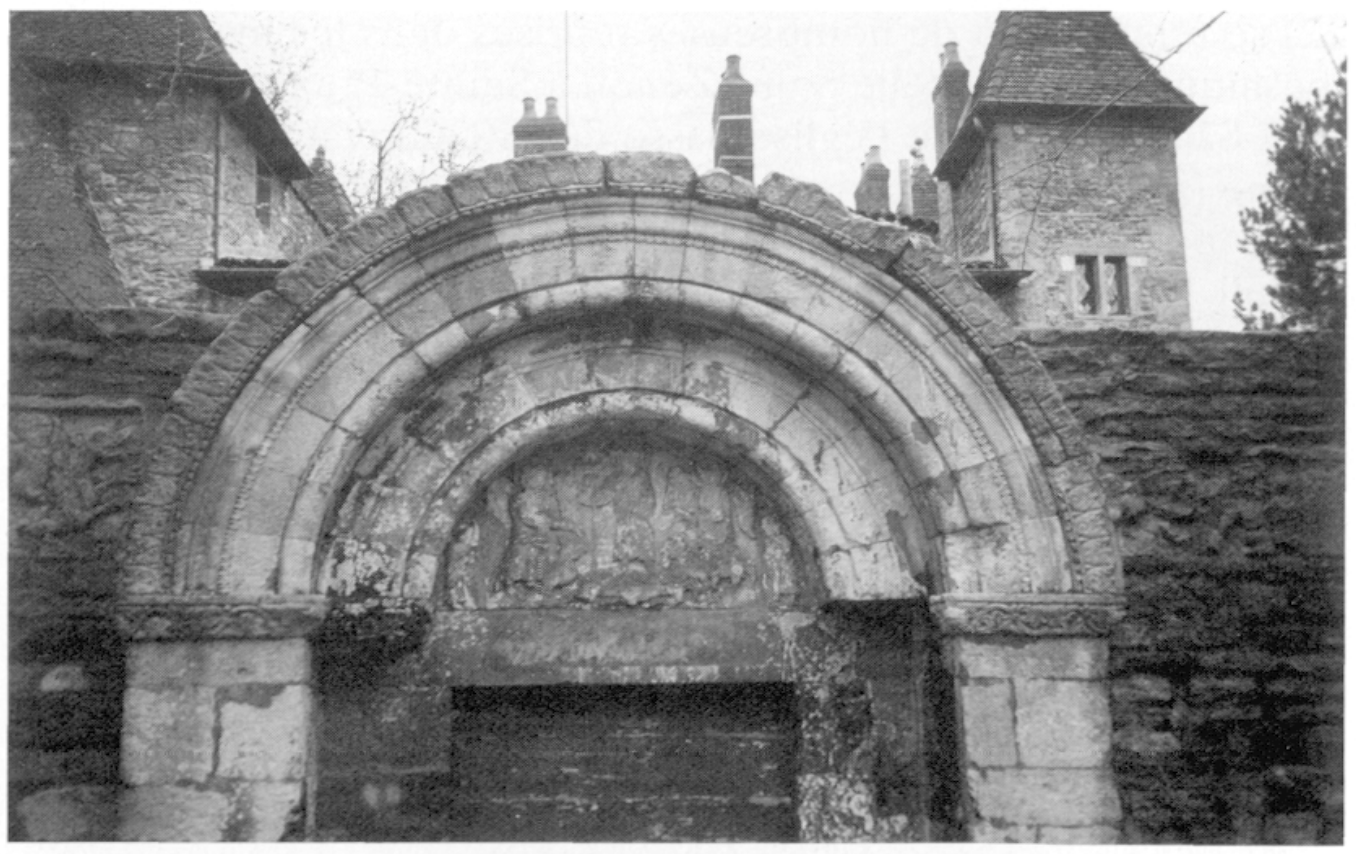

FIG. 2. - Le tympan roman. Cl. R. Favreau. 
$\mathrm{XXX}^{\mathrm{e}}$ Légion, dans un encadrement circulaire, surmontée des deux figures emblématiques du Rhône et de la Saône avec des urnes d'où s'écoule de l'eau :

« Aux dieux mânes et à la mémoire éternelle de Casius Annius Flavianus, vétéran de la trentième légion, Annius Respectus et Julia Restituta, son fils et sa femme... $»^{5}$.

Sur le panneau qui suit sont personnifiées les saisons, le Printemps avec un panier de fleurs, l'Été avec un panier d'épis, l'Automne avec un panier de raisins et une serpe, l'Hiver tenant un lièvre par les pattes et se couvrant la tête d'un voile.

Au tympan de la porte romane (fig. 2) du réfectoire de l'îleBarbe, le Christ est représenté foulant aux pieds un lion et un dragon ; il est entouré de deux anges qui écrasent un aspic et un basilic. De chaque côté de la tête du Christ, on pouvait lire :

EGO SUM LUX MUNDI

« Je suis la lumière du monde ».

Une inscription de trois vers sur la première archivolte (fig. 3) commente la scène. Les deux vers gravés sur la seconde archivolte (fig. 4 et 5) sont consacrés au Créateur et au Sauveur.

L'affirmation du Christ «Je suis la lumière du monde » (Jn 8, 12) se rencontre à de nombreuses reprises dans les inscriptions : mosaïque de la chapelle Saint-Zénon à Sainte-Praxède de Rome (817-824), fresque de l'église basse des SS. Giovanni e Paolo de Rome ( $\mathrm{IX}^{\mathrm{e}}-\mathrm{X}^{\mathrm{e}} \mathrm{S}$.), trésor de la cathédrale de Milan $\left(\mathrm{XI}^{\mathrm{e}} \mathrm{s}\right.$.), tympan de la porte sud de Saint-Aventin, abside de San Clemente de Tahull, bâtiments abbatiaux de Saint-Maximin de Trèves (XII ${ }^{\mathrm{e}} \mathrm{s}$ ), Panteón de los Reyes de San Isidoro de León (vers 1180), mosaiqque de l'abside de Monreale (1186), crypte de la cathédrale d'Anagni, peintures de l'abside romane de Tavant, chapiteau du chœur de Saint-Martin d'Ainay à Lyon, devant d'autel de la cathédrale de Marseille ou de l'église de Saint-Genis-des-

5. Abbé Roux, op. cit. (n. 1), p. 78, L. Niepce, op. cit. (n. 1), p. 15 :

D.M.

Et memoria

æternæ C. Anni

Flaviani Vet. Ex leg. XXX

Annius Respectus et Julia

Restituta, filius et conjux,

Qua..... v........ 


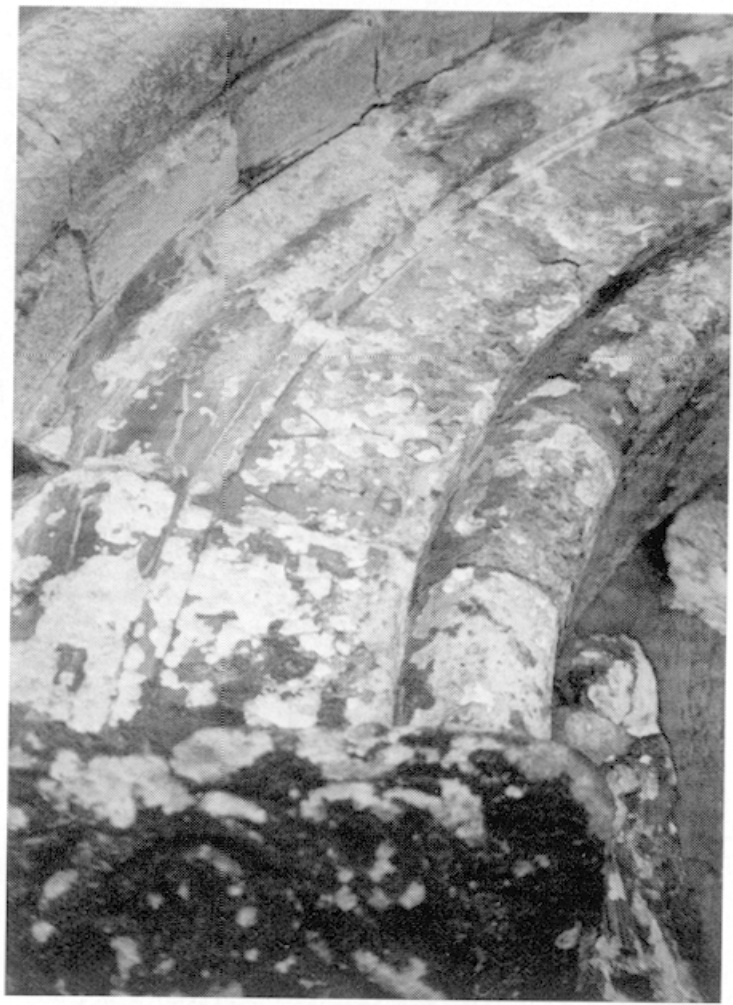

FIG. 3. - Détail de la première archivolte : inscription sur trois lignes. Cl. R. Favreau.

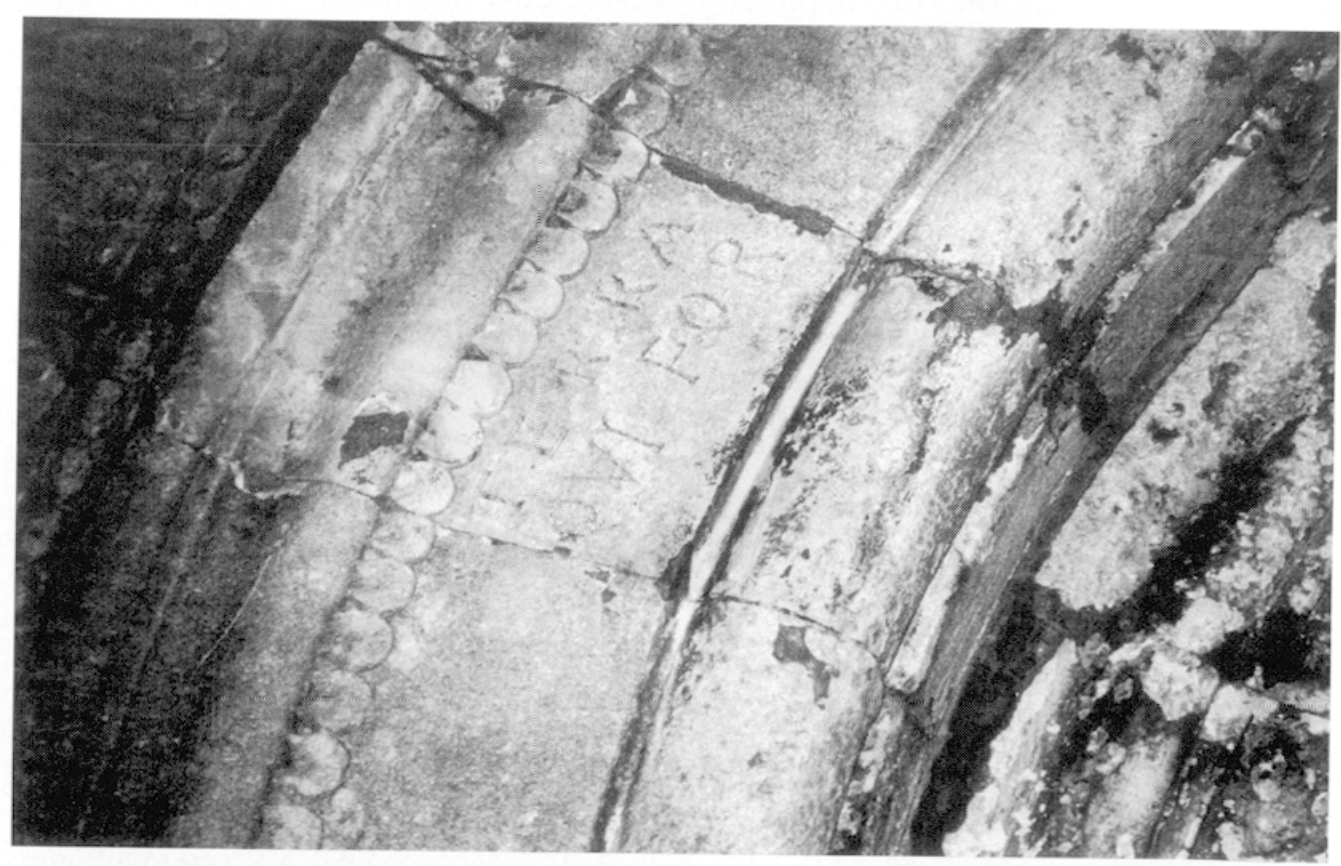

FIG. 4. - Détail de la seconde archivolte, côté gauche. Cl. R. Favreau. 
Fontaines, façade de la cathédrale de Modène ${ }^{6}$. Sur le livre du Christ au cul-de-four de l'abside d'Indensen en Saxe on peut lire la totalité du verset de Jean :

EGO SUM LUX MUNDI, QUI SEQUITUR ME NON AMBULAT IN TENEBRIS SED HABEBIT LUMEN VITE ${ }^{7}$.

Les moines qui entraient chaque jour au réfectoire devaient comprendre que le Christ est lumière du monde et, en poursuivant le verset assurément pour eux familier, que celui qui le suit ne marche pas dans les ténèbres. Les ténèbres, ce sont les puissances du mal que, précisément, le Christ et ses anges foulent aux pieds.

Deux des trois vers de la première archivolte commentent l'iconographie du tympan :

ASPIS CALCATUR BASILISCUS SED SUPERATUR
SICQUE LEO PARITERQUE DRACO SUNT MISTICA VERO

«L'aspic est foulé aux pieds, et le basilic est terrassé

et ainsi le lion et également le dragon, ils sont vraiment des animaux

[mystiques ».

La mise en vers du verset 13 du psaume 91 (90) :

Super aspidem et basiliscum ambulabis et conculcabis leonem et draconem

«Tu marcheras sur l'aspic et le basilic et tu fouleras aux pieds le lion et le dragon »,

est laborieuse : emploi du sed au premier vers, double enclitique -que et adjonction d'un vero dans le second vers. On a pourtant réussi à faire au départ un vers léonin riche. Ce qui est le plus intéressant, c'est qu'on a tenu à indiquer qu'il fallait de la scène, faire une interprétation «mystique », c'est-à-dire allégorique ${ }^{8}$.

6. T. Dobrzeniecki, «Majestas Domini... ", Rocznick Muzeum Narodowego w Warszawie XIX (1975), p. 235 ; Lanfranco e Wiligelmo. Il duomo di Modena, Modène, 1984, p. 383 ; R. Favreau, J. Michaud et B. Mora (éd.), Corpıss des inscriptions de la France médiévale, t. 14, Paris, 1989, p. 95-96.

7. W. Wulf, Saxe romane, La Pierre-qui-Vire, photographie en face de la page 300.

8. Ainsi une incription du flabellum de Kremsmunster (milieu du XII ${ }^{\mathrm{e}}$ siècle) nous avertit-elle que le lion représenté est un lion «mystique » (mysticus ecce leo) qui ressuscite (surgit). Sur un émail de la fin du $\mathrm{XII}^{\mathrm{e}}$ siècle au Musée Condé à Chantilly les offrandes de Melchisédech sont dites « mystiques » et les deux bois réunis par la veuve de Sarepta sont des mistica signa. des signes mystiques de la croix (M.-M. Gauthier, Emaux du Moyen Âge occidental. Fribourg, 1972,p. 356). Dans le retable de Nicolas de Verdun à Klosterneubourg, on trouve employé le verbe misticat et l'adjectif mistica. 


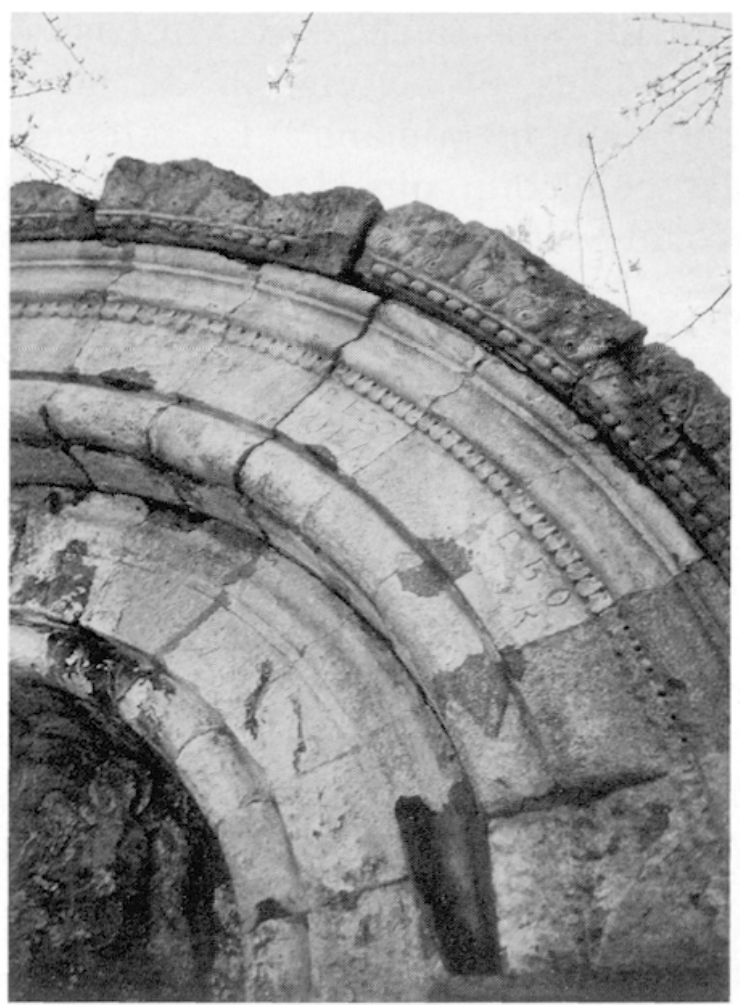

Fig. 5. - Détail de la seconde archivolte, côté droit. Cl. R. Favreau.

Les premiers exemples d'une représentation du Christ marchant sur les quatre animaux du psaume 91 se trouvent sur des sarcophages espagnols du $\mathrm{IV}^{\mathrm{c}}$ siècle. On a pensé que le tympan de l'île-Barbe aurait son inspiration dans une miniature du XI ${ }^{\mathrm{e}}$ siècle d'un manuscrit d'Ensiedeln, où le Christ foulant aux pieds le lion et le dragon est entouré de deux petits personnages, Heto et Adelheit (les copistes ?) qui reçoivent sa bénédiction'. Et il est vrai qu'au tympan de l'île-Barbe on distingue encore bien, à côté de chaque ange, un petit personnage agenouillé, sans doute ici des moines. Le verset 14 du psaume 91 (90) est d'ailleurs inscrit auprès du Christ dans le manuscrit d'Einsiedeln.

Ce thème est fréquemment représenté. Le verset $13 \mathrm{du}$ psaume 91 (90) est cité sur une plaque d'ivoire de 800 environ à la Bodleian Library d'Oxford ${ }^{10}$, sur le diptyque de Genoels-

9. V. Terret, op. cit. (n. 2), p. 28.

10. Le Monde de la Bible, hors-série, Charlemagne un empereur chrétien pour l'Europe, automne 2004, p. 54 (SUPER ASPIDEM). 


\section{COMPTES RENDUS DE L'ACADÉMIE DES INSCRIPTIONS}

Elderen de la fin du viII ${ }^{e}$ siècle, aux Musées royaux d'Art et d'Histoire de Bruxelles, et au pignon de la châsse de saint Hadelin à Visé à l'époque romane ${ }^{11}$. La référence est explicite mais le sens est beaucoup plus large dans l'inscription d'un diptyque d'ivoire au Museo nazionale del Bargello de Florence :

QUO MORS EST STRATA SERPENTE LEONE NOTATA

REX DEUS ESI ET HOMO QUEM SIMPLEX SIGNAT IMAGO ${ }^{12}$

«C'est la mort qui est notée par le terrassement du serpent et du lion.

Le roi est Dieu et homme, lui que désigne une simple image ».

Le verset du psaume est exactement cité au $\mathrm{XII}^{\mathrm{e}}$ siècle à la porta regia de la cathédrale de Modène et sur un bas-relief du Christ à Notre-Dame de Maastricht ${ }^{13}$. Il ne l'est que partiellement sur la châsse de saint Éleuthère (1247) à la cathédrale de Tournai (CONCULCABIS LEONEM ET DRACONEM) ${ }^{14}$ et il est intégré dans un distique sur une mosaïque de l'abside sud de Saint-Just de Trieste au XIII ${ }^{\mathrm{e}}$ siècle $^{15}$ :

MAJESTATE DEUM LIQUET HUNC REGNARE PER $Æ V U M$

AMBULAT EN CHRISTUS SUPER ASPIDEM ET BASILISCUM

Le thème et la citation du psaume se rencontrent aussi dans les manuscrits, ainsi à Cambridge ou à Munich ${ }^{16}$.

Tous les commentateurs de ce psaume 91 (90) tiennent le même discours : ces quatre animaux représentent le diable. Augustin est la source principale de tous les commentaires dans

11. Rhin-Meuse. Art et civilisation 800-1400, Bruxelles-Cologne, 1972, p. 163 : UBI DOMINUS AMBULAVIT SUPER ASPIDEM ET BASILISCUM ET CONCULCABIT LEONEM ET DRACONEM (Genoels-Elderen); DOMINUS POTENS IN PRELIO BELLIGER INSIGNIS TIBI, SIC BASILISCUS ET ASPIS SUBDOLUS ATQUE LEO SUBEUNT REX IN CRUCE PASSO (Visé).

12. J. Beckwitt, Ivory Carvings in Early Medieval England, Londres, 1972, p. 136, $\mathrm{n}^{\circ} 85$. Le mot strata étant un ablatif ( $a$ final long) doit se rattacher à serpente.

13. A.K. Porter, Lombard Architecture III, New York-Londres-Oxford, 1917, p. 43-44 ; L. Tollenaere, La sculpture sur pierre dans l'ancien diocèse de Liège à l'époque romane, Gembloux, 1957, p. 274, pl. $20^{A}$.

14. S. Collon-Gevaert, Histoire des arts du métal en Belgique, Bruxelles, 1951, p. 220 , pl. 44.

15. Ph. Verdier, op. cit. (n. 2), p. 86, n. 4.

16. K.M. Openshauw, "The Symbolic Illustration of the Psalter : an Insular Tradition », Arte medievale 1992-1991, p. 52, fig. 16 (Cambridge University Library, ms. Ff. I 23, fol. $195 v^{\circ}$ ); Ph. Verdier, op. cit (n. 2), p. 73, fig. 48 (Bayerische Staatsbibliothek, Clm 14159. fol. $\left.5 v^{\circ}\right)$. 
son Enarratio in psalmum $X C^{17}$ : il stigmatise les tromperies du serpent, allusion évidente à la Genèse, la cruauté du lion qui dévore les martyrs, les pièges du dragon qui évoquent les hérésies, et il déclare le basilic « le roi des serpents comme le diable est le roi des démons ", référence continuellement reprise en raison du rapprochement entre basilicus et basileus, «l'empereur ». Sedulius rappelle que le Christ nous a rachetés en écrasant sous ses pieds le lion et le dragon ${ }^{18}$. Pour Cassiodore, aspic, basilic, lion, dragon, «tous ces noms conviennent parfaitement au diable ", tous seront jetés à terre sous les pieds du Seigneur lors de son glorieux avènement ${ }^{19}$. Le pseudo-Bède le Vénérable, Haymon d'Halberstadt, Rémi d'Auxerre, la Glose ordinaire ${ }^{20}$ reprennent le commentaire d'Augustin. Haymon, évêque d'Halberstadt, précise que «par le dragon il faut entendre le diable... sur lequel le Christ a marché lorsque, par sa mort, il a détruit son pouvoir ». Brunon, évêque de Würzburg, copie littéralement Cassiodore $^{21}$. Brunon Segni, a une exégèse voisine de celle d'Haymon ${ }^{22}$. Oddon, moine d'Asti, ajoute que dans le baptême le diable est terrassé ${ }^{23}$. Pierre Lombard copie Cassiodore ${ }^{24}$, Gerhoh, prévôt de Reichersperg ${ }^{25}$, a un commentaire plus développé. Il fait référence au Nouveau Testament : Jésus qui vient à bout des deux démoniaques gadaréniens (Mt 8,29), qui donne aux apôtres «le pouvoir de fouler aux pieds serpents, scorpions et toute puissance de l'Ennemi » (Lc 10,18), Paul qui écrit aux Romains que le Dieu de la paix écrasera bien vite Satan sous leurs pieds $(16,20)$. Il identifie l'aspic à la mort, le basilic au péché, le lion à l'Antéchrist, le dragon au diable, et conclut que le Christ l'a emporté - superavit, le mot employé à l'île-Barbe - sur l'aspic et le basilic, et qu'au jour du jugement il précipitera dans le feu l'Antéchrist et le diable. On trouve encore chez Jean Beleth, une allusion au lion et au dragon ${ }^{26}$.

17. Patrologie latine, t. 37, c. 1168.

18. Ibid., t. 19, c. 769-770.

19. Ibid., t. 70, c. 654 .

20. Ibid., t. 93, c. 976 (le De psalmorum libro exegesis oublié sous le nom de Bède serait de Mangold de Lautenbach) ; t. 116, c. 511 (Haymon) ; t. 131, c. 639 (Rémi) ; t. 113, c. 1000 (Glossa ordinaria).

21. Ibid., t. 142 , c. 3340

22. Ibid., t. 164, c. 1055 .

23. Ibid., t. 165 , c. 1259.

24. Ibid., t. 191, c. 853 .

25. Ibid. t. 194, c. 560-561.

26. Ibid.. t. 202, c. 130 . 
On trouve dans les bestiaires des commentaires de même ordre sur l'aspic, le basilic, le dragon, commentaires qui s'inspirent, voire copient littéralement, les Etymologies d'Isidore de Séville, ou parfois se rapprochent des commentaires sur le psaume 90. L'exemple de Raban Maur est intéressant, en ce sens qu'il copie littéralement Isidore de Séville pour l'aspic et le dragon, et Cassiodore pour les serpents ${ }^{27}$. Pour le dragon, il écrit :

«Mystiquement (mystice), le dragon signifie ou le diable ou ses ministres, ou les persécuteurs de l'Église ».

Il est toutefois un point sur lequel bestiaires et commentaires du psaume divergent : dans les bestiaires le lion est toujours représenté comme un animal positif, car «le lion est le plus fort des animaux » (Proverbes 30,30), et il est selon Raban Maur la figure même du Christ. Aussi le tympan de la cathédrale de Jaca qui illustre le psaume 90 mais en même temps glorifie la puissance du Christ sous la figure du lion, a représenté le Christ vainqueur de la mort sous les traits d'un lion qui écrase un ours et un basilic ${ }^{28}$.

Les deux vers léonins riches de la seconde archivolte de la porte romane de l'Île-Barbe sont d'une bonne veine poétique et présentent deux aspects différents de la figure du Christ. En foulant les quatre animaux maléfiques, il écrasait les puissances du mal. Cette fois, il est dit le Créateur : tous les éléments lui sont soumis, et le Sauveur : sur la croix il détruit la mort.

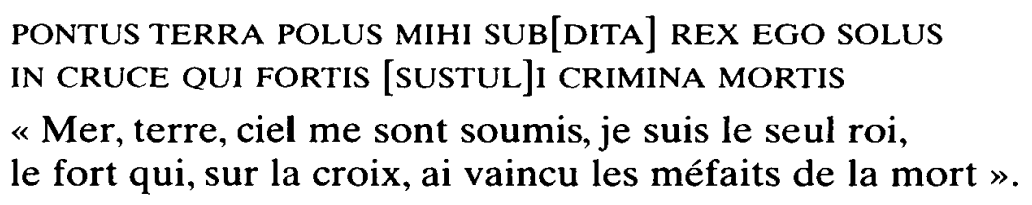

Le premier vers fait partie d'un distique d'un poème attribué à Hildebert de Lavardin et intitulé Christus de se ipso « ce que dit le Christ de lui-même ». Le second vers d'Hildebert est aussi consacré au Dieu créateur :

SOLUS AB ETERNO CREO CUNCTA CUNCTA GUBERNO

«Seul, de toute éternité, je crée tout, je gouverne tout ${ }^{29}$.

27. Ibid., t. 111 (De universo libri $X X I I$ ), c. 230 (aspic), 229 (dragon), 231 (serpent). p. 541.

28. R. Favreau, «Les inscriptions du tympan de la cathédrale de Jaca ", CRAI 1996,

29. Patrologie latine, t. 171, c. 1436. 
Les deux vers, à un mot près - rego au lieu de ego au premier vers - sont inscrits sur la châsse de Notre-Dame, du premier tiers du XIII ${ }^{\mathrm{e}}$ siècle, au trésor de la cathédrale d'Aix-la-Chapelle ${ }^{30}$. Le second vers, absent de l'île-Barbe, figure, avec le Christ sur un des petits côtés de la châsse de saint Remacle, de 1268 , à Stavelot ${ }^{31}$. La critique, aujourd'hui, rejette ces deux vers des œuvres authentiques d'Hildebert, mais à l'époque on les pensait de lui, et il est, au XII ${ }^{\mathrm{e}}$ siècle, le versificateur de référence. Ce distique figure aussi dans un manuscrit d'Oxford ${ }^{32}$.

Quant au second vers inscrit à l'île-Barbe il évoque la croix, in cruce, mais aussi la victoire, fortis, sur la mort, crimina mortis, cette dernière expression se trouvant dans Virgile ${ }^{33}$. On peut citer un poème de Froumundus qui a un sens très voisin :

Ecce leo fortis transit discrimina mortis ${ }^{34}$.

Surtout l'expression leo fortis est associée à la figure du Christ de la Résurrection, grâce à une des caractéristiques données au lion par les bestiaires ${ }^{35}$, de façon très fréquente tant chez les exégètes que les poètes, dans les hymnes, les tropes, dans de nombreuses inscriptions, au point que le mot fortis à lui seul arrive à évoquer la victoire du Christ sur la mort dans sa Passion et sa Résurrection ${ }^{36}$.

Reste un dernier vers qui se trouve sur la première archivolte entre les deux distiques ci-dessus présentés :

ALFA VEL O PRIMUS FINIS MIHI CONVENIT ERGO

«Alpha et omega, le début et la fin me conviennent donc ».

30. Die Inschriften des Aachener Doms, éd. Helga Giersiepen. Wiesbaden, 1992 (Düsseldorf Reihe, 1), $\mathrm{n}^{\circ} 35$, p. 37-39.

31. A. de Montaiglon, "De quelques inscriptions en vers", Revue de l'art chrétien, $33^{\circ}$ année, 1880 , p. 9 ; J. Herbig. L'art mosan depuis l'introduction du christianisme jusqu'à la fin du XVIIr siècle. I. Des origines à la fin du Xve siècle, Bruxelles, 1906, planche en vis-à-vis de la page 52.

32. D'après Hans Walther, Carmina medii avvi posterioris latina. II. Proverbia sententiaque latinitatis medii avi, 3, Göttingen, $1965, \mathrm{n}^{\circ} 21930 \mathrm{a}, \mathrm{p} .884$. Walther cite aussi le premier vers dans I. Initia carminum ac versuum medii aevi posterioris latinorum, Göttingen, $1959, \mathrm{n}^{\circ} 14268$, p. 738.

33. Enéide, VI, v. 430 : hos juxta falsos damnati crimina mortis.

34. K. Strecker, N. Fickermann, G. Silagi et B. Bischoff (éd.), Die lateinischen Dichter des deustchen Mittelalters. IV. Die Ottonenzeit, Leipzig-Berlin-Munich. 1937-1979, p. 435.

35. D'après les bestiaires, le lion, le troisième jour après leur naissance, souffle sur ses lionceaux, jusque-là inanimés, et les éveille à la vie.

36. R. Favreau, "Le thème iconographique du lion dans les inscriptions médiévales ". CRAI 1991, p. 613-636. 


\section{COMPTES RENDUS DE L'ACADÉMIE DES INSCRIPTIONS}

La citation biblique est facile à identifier. L'inscription fait référence à l'Apocalypse, 1,8 et $17 ; 21,6$ et 22,13 , dont on a une annonce dans Isaïe 41,$4 ; 44,6$ et 48,12 . La versification n'est pas d'un grand poète, mais le texte s'y prêtait mal. On a plutôt retenu dans les inscriptions une citation exacte de l'Apocalypse, à des dizaines de reprises avec les seuls alpha et omega, parfois plus complètement tels le EGO SUM A ET O PRIMUS ET NOVISSIMUS de Sant'Angelo in Formis ou le EGO SUM A $\Omega$ PRINCIPUM ET FINIS de la croix de l'abbayes de Clairmarais à Notre-Dame de SaintOmer.

Le sens principal est donné par l'iconographie du tympan, c'est-à-dire la figure du Sauveur écrasant le lion et le dragon, tandis que ses anges marchent sur l'aspic et le basilic. Dans ce milieu monastique, cette illustration du psaume 91 (90) aura sûrement renvoyé aux tentations du Christ par Satan dans le désert, au début de sa vie publique, car les versets 11-13 du psaume constituent une antienne et un répons du premier dimanche de Carême ${ }^{37}$, et l'évangile de ce premier dimanche est celui des Tentations du Christ au désert (Mt 4, 1-11), qui se termine par la déroute du diable, tandis que «les anges s'approchent et servent le Christ ». Dans son traité «Sur la prière », Évagre le Pontique $(† 395)$ raconte que le «Malin livra une telle guerre à l'un des saints lorsqu'il était en prière qu'à peine celui-ci avait-il étendu les bras, que le démon se changeait en lion » et l'assaillait, et qu'il en fut de même pour le très illustre moine Jean le Petit que le démon s'efforçait de troubler «sous la forme d'un dragon ${ }^{38}$. Pour les premiers moines chrétiens le désert fourmillait d'êtres maléfiques sous la forme de lions, ours, léopards, taureaux, loups, satyres, hiboux, serpents, scorpions ${ }^{39}$. Sur l'une des faces de la croix de Ruthwell en Écosse, du début du VIII ${ }^{\mathrm{e}}$ siècle, le Christ foule deux animaux et l'inscription dit que «bêtes et dragons reconnurent au désert le Sauveur du Monde ${ }^{40}$. Saint Bernard a

37. J.-R. Hesbert (éd.), Corpus antiphonalium officii, t. IV, Rome, 1970 (Rerum ecclesiaticarum documenta, series major, fontes, 10), $\mathrm{n}^{\circ}$ 6987, p. 21.

38. Évagre le Pontique, De la prière à la perfection. "Sur la prière », traduit par M.-O. Goudet, Paris, 1992 («Les Pères dans la Foi »), p. 96, \$106 et 107.

39. Chr. Cannuyer, « Du désert des pharaons au désert des anachorètes ", Le Monde de la Bible, n 116 (1999), p. 28.

40. E. Okasha, Hand-List of Anglo-Saxon Non-Runic Inscriptions, Cambridge, 1971. $\mathrm{n}^{\circ} 105$, p. 108-112 ; Fr. Sharratt et P. Sharratt, Écosse romane, La Pierre-qui-Vire, 1985. p. 33. fig. 2 : COGNOVERUNT IN DESERTO SALVATOREM MUNDI. 
consacré, à l'époque du tympan de l'île-Barbe, 17 sermons de Carême au psaume 91 (90). Dans son $13^{e}$ sermon il identifie l'aspic à la surdité, le basilic à l'envie, le dragon à la colère, le lion à la crainte $\mathrm{c}^{41}$. Dans le $14^{\mathrm{e}}$ sermon consacré au verset super aspidem et basiliscum, il propose les quatre vertus cardinales, la force, la prudence, la tempérance, la justice à opposer aux quatre tentations dont les quatre animaux sont la figure ${ }^{42}$. C'est à propos du dimanche des Rameaux qu'Honorius Augustodunensis, exact contemporain de Bernard de Clairvaux, commente le psaume 91 (90). L'aspic figure le péché qui rend sourd aux enseignements du Christ, le basilic désigne la mort, le lion représente l'Antéchrist, le dragon est le diable, roi de tous les maux. «Le Seigneur a marché sur l'aspic et le basilic lorsqu'il a, par sa mort, détruit le péché et la mort, il foulera aux pieds le lion et le dragon lorsqu'il l'emportera sur l'Antéchrist et condamnera au dernier jugement le diable et ses suppôts ${ }^{43}$. Tout ce contexte évoqué par le psaume devait être familier aux moines de l'île-Barbe.

Le Ego sum lux mundi inscrit autour du Christ est une antienne du dimanche de la Passion ${ }^{44}$. Il faut y joindre le distique où le Christ est dit le maître des éléments et le vainqueur de la mort par sa croix et sa Résurrection.

Enfin le verset de l'Apocalypse inscrit sur le tympan figure lui aussi dans la liturgie et forme une antienne du dimanche de Pâques ${ }^{45}$. Désormais le Christ est en gloire dans le ciel, comme le dira l'Apocalypse.

Carême, Passion et Résurrection, gloire du Christ après l'Ascension, telle semble être la lecture d'ensemble de la porte romane de l'île-Barbe, à partir des inscriptions qui accompagnent la figure du Christ foulant aux pieds les quatre animaux du psaume 91 (90). Ce n'est pas, en tout cas, une iconographie et une épigraphie qui s'imposaient pour un réfectoire. Mais y avait-il des images et des textes à retenir préférentiellement pour un réfectoire?

Les inscriptions n'ont pas dû être exceptionnelles dans les réfectoires monastiques. Déjà au $\mathrm{VI}^{\mathrm{e}}$ siècle saint Martin de Braga

41. Patrologie latine, t. 183 , c. 236-238.

42. Ibid., c. 240-243.

43. Ibid., t. 172, c. 915-916.

44. J.-R. Hesbert, Corpus antiphonalium officii, t. III, Rome, 1970 (Rerum ecclesiaticarum documenta, series major, fontes, 9), p. 195.

45. Ibid., t. III, p. 194-195. 
compose cinq distiques élégiaques à inscrire in refectorio, dans ou sur un réfectoire. Il y souligne que l'on n'y trouvera pas des lits de table, des mets brillants, des vins précieux, mais que la grâce du Seigneur viendra y suppléer la simplicité de la table ${ }^{46}$. Alcuin, à la fin du virre siècle, rédige à son tour treize vers « pour le réfectoire des moines ", à l'intention de la communauté de Saint-Hilaire-leGrand de Poitiers : que le Christ bénisse les mets de cette table, que les convives le louent sans cesse, que leurs mains soient largement ouvertes aux pauvres ${ }^{47}$. Des inscriptions accompagnaient le lavabo situé à l'entrée des réfectoires. A Saint-Denis était souligné le rôle de l'abbé Hugues dans l'établissement d'un magnifique bassin circulaire d'où l'eau jaillissait par vingt-huit ouvertures $^{48}$. A Saint-Martin d'Ainay à Lyon, un distique disait que «sous le toucher angélique la piscine de Siloé s'agite; le premier malade qui va s'y laver est guéri ", référence à Jean 5 , 2-5, mais confusion de la piscine de Siloé avec la piscine de Bézatha où l'aveugle va, sur l'ordre de Jésus, se laver et est guéri (Jean 9, 6-7 $)^{49}$. A Saint-Honorat de Lérins, le lavabo existe toujours et est déposé dans le cloître. L'inscription dit :

«Ô Christ, de ta dextre qui purifie et le dedans et le dehors, purifie plus intérieurement ce que cette onde ne peut purifier $\aleph^{50}$.

Dans le même ordre d'un rapport direct de l'inscription avec la fonction d'un réfectoire, on peut citer le tympan du réfectoire de l'abbatiale de chanoines réguliers de l'ordre de Prémontré à Rothenkirchendorf, d'environ 1200 :

SEDIBUS HIS PANEM CARNI VERBUM DABIS AURI

DELICIIS VERBI SACIUS QUAM PANE CIBARIS

46. Patrologie latine, t. 72, c. 52 . Ce poème iconographique figure dans le manuscrit latin 2832, fol. $117 \mathrm{v}^{\circ}-118$ de la Bibliothèque nationale de France.

47. E. Duemmler (éd.), Pata latini avi carolini, Berlin, 1881 (Monumenta Germania Historica. Pcetarum latinorum Medii AEvi, t. 1), p. 327-328. R. Favreau et J. Michaud (éd.), Corpus des inscriptions de la France médiévale 1, Paris-Poitiers, 1974, p. 56-57 (édition et traduction).

48. F. de Guilhermy, Inscriptions de la France du ve siècle au xvIIr siècle, t. II, Ancien diocèse de Paris. Paris, p. 149 :

HUGONI FRATRES ABBATI RFDDITE GRATES HOC MANIBUS FRATRUM NAM SUSTULIT ISTE I.AVACRUM

Il s'agit de Hugues 11, abbé de 1197 à 1204.

49. R. Favreau, J. Michaud, B. Mora (éd.), op. cit. (n. 3), p. 92.

50. R. Favreau, J. Michaud, B. Mora (éd.), op. cit. (n. 6), p. 7-8. 
«Dans ce lieu tu donneras au corps le pain et à l'oreille la parole, mais tu seras mieux nourri par les joies de la Parole que par le pain $»^{51}$.

La référence est claire à la lecture à haute voix pendant que les chanoines réguliers prenaient les repas.

On peut mettre d'autres inscriptions en vis-à-vis de cette première catégorie de textes. A Saint-Maximin de Trèves l'inscription de la porte du réfectoire d'été disait :

LUX EGO SUM MUNDI COELESTIS JANUA VITAE

QUI ME DILIGITIS AD GAUDIA PLENA VENITE

SIT PAX INTRANTI PAX VERA SIT EGREDIENTI EGO SUM RESURRECTIO ET VITA ${ }^{52}$

« Je suis la lumière du monde, la porte de la vie céleste, vous qui m'aimez venez aux joies en plénitude.

Que la paix accompagne celui qui entre, et la vraie paix celui qui sort. Je suis la résurrection et la vie».

On notera qu'on a ici la même citation de Jean 8,12 , « Je suis la lumière du monde » qu'à l'île-Barbe. Le janua vita, qu'emploient Bède, Alcuin, Raban Maur, Marbode ou Baudri de Bourgueil se retrouve au portail de San Giorgio in Palazzo à Milan, à celui de Saint-Marcel-lès-Sauzet dans la Drôme, au tympan de San Pablo del Campo à Barcelone, au tympan de Jaxa à Wrocław ou encore au narthex peint de l'abbatiale de Payerne. Le Sit pax intranti se lit à Villemartin de Sotoscueva en Castille, à Roda, à Dompeter en Alsace, à Saint-Léger-en-Pons, en Saintonge, à Cingoli, Spolète, Saint-Apollinaire et Saint-Blaise à Rome, et la forme Pax intranti est déjà aux premiers siècles chrétiens à SidiFerruch en Algérie ${ }^{53}$. Bref on a davantage à faire ici avec le thème de la porte en général qu'avec la porte d'un réfectoire en particulier.

Dans l'ancienne abbaye de Saint-Aubin, à Angers, la porte romane du réfectoire est remarquablement conservée. Deux inscriptions illustrent les scènes : vertus écrasant les vices, agneau

51. D. von Winterfeld, Palatinat roman, La Pierre-qui-Vire, 1993, p. 324.

52. F. X. Kraus, Die christlichen Inschriften der Rheinlande von der Mitte des Achten bis zur Mitte des Dreizehnten Jahrhunderts, Fribourg-en-Brisgau - Leipzig, 1894, n ${ }^{\circ}$ 372, p. 179. Il y avait aussi une inscription sur la porte du réfectoire d'hiver.

53. R. Favreau, "Le thème iconographique de la porte ", Cahiers de Civilisation médiévale 34 (1991), p. 267-279. 
encensé par des anges, Samson, figure du Christ, écrasant le lion ${ }^{54}$. Bien que l'iconographie soit très différente de celle de l'ÎleBarbe, on pouvait $\mathrm{y}$ trouver un même thème général. Mais pas plus qu'à l'île-Barbe ne s'impose un rapport rigoureux avec le réfectoire. L'exemple du réfectoire de Saint-Benoît-sur-Loire où, au début $\mathrm{du} \mathrm{XI}^{\mathrm{e}}$ siècle, on avait représenté les fables de Phèdre (imitées d'Ésope), accompagnées de sentences morales, et le Dieu de l'Apocalypse entouré des vingt-quatre vieillards ${ }^{55}$, invite aussi à considérer qu'il n'y a pas de programme contraignant pour l'iconographie et les inscriptions qui se rapportent à un réfectoire.

La vie du moine, comme celle des fidèles, se résume en un combat pour qu'en lui les forces du bien l'emportent sur les forces du mal. Les moines de l'île-Barbe pouvaient se le remémorer chaque fois qu'ils se rendaient à leur réfectoire, en voyant le Christ écraser l'aspic et le basilic, le lion et le dragon, le Christ qui par sa croix a vaincu la mort et les attend dans sa gloire en la Jérusalem céleste. A défaut d'être directement liées à la fonction $\mathrm{du}$ réfectoire, l'iconographie et les inscriptions de la porte romane de l'île-Barbe offraient aux moines une leçon essentielle. Et ici, bien plus qu'un simple commentaire, les inscriptions sont un complément indispensable pour la compréhension générale.

Reste que, pour l'étude des inscriptions, s'impose régulièrement une recherche des sources, expressions ou vers empruntés à des auteurs classiques ou chrétiens, formules, citations bibliques, liturgiques, hagiographiques, reprises de textes inscrits sur d'autres monuments... Déjà en 1894 Léon Germain appelait l'attention sur «l'utilité d'un recueil des formules épigraphiques chrétiennes... accompagné de renseignements précis de chronologie et d'origine ${ }^{56}$. Un tel recueil des citations, formules, expressions est en cours à Poitiers pour les inscriptions des $\mathrm{VIII}^{\mathrm{e}}$ $\mathrm{XIII}^{\mathrm{e}}$ siècles. J'ai terminé ce travail, en cours de révision à la date de cette publication. Est déjà acquis le concours des collègues épigraphistes allemands, autrichiens, italiens, espagnols. Lorsque le logiciel en cours d'étude aura été mis au point, alors ce réper-

54. P. d'Herbécourt, Anjou roman, La Pierre-qui-Vire, 1959, p. 175.

55. R.-H. Bautier, «Le monastère et les églises de Fleury», dans les Mémoires de la Société nationale des antiquaires de France, $9^{\text {e }}$ série, t. IV, 1969, p. 138-146; L. Herrmann, «Autour des fables de Phèdre ", Latomus VIII (1949), p. 203-207.

56. Revue de l'art chrétien, $37^{\mathrm{e}}$ année, 1894, p. 138. 
toire pourra être immédiatement utile à tous les médiévistes, et être régulièrement enrichi par de nouvelles inscriptions ou par de nouvelles recherches.

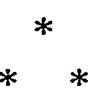

MM. Marc Philonenko, Henri Lavagne, correspondant de l'Académie, Michel ZINK et Pierre-Yves Lambert, correspondant de l'Académie, interviennent après cette communication. 


\title{
APPENDICE
}

Le Gallia christiana, IV, c. $225,227,229$, et Léopold Niepce, dans son étude sur L'Île-Barbe (op. cit. [n. 1]), p. 214, 244, 252, 267, ont donné, d'après le prieur du XVII ${ }^{\mathrm{e}}$ siècle Claude Le Laboureur, quatre épitaphes qui existaient encore au $\mathrm{XVII}^{\mathrm{e}}$ siècle.

I. Dans la salle capitulaire, sur le mur de séparation avec l'aile droite de l'église abbatiale, était peinte l'épitaphe de l'abbé Humbert, élu en 1032, mort en 1056 :

HIC PATER HUMBERTUS MONACHORUM GLORIA VIXIT, QUI SOBRIUS.

«Ici a vécu l'abbé Humbert, gloire des moines, qui [fut] sobre... »

II. Dans l'abbatiale Saint-Martin-et-Saint-Loup se trouvait la tombe de l'abbé Gaucerand (1198) devenu en 1200 évêque de Saint-Paul-Trois-Châteaux :

\author{
VI IDUS MAII DEPOSITIO \\ DOMINI GAUCERANNI TRICASTINENSIS EPISCOPI \\ ET ABBATIS INSULAE BARBARAE CUJUS \\ ANIMA REQUIESCAT IN PACE
}

« Le 6 des ides de mai (10 mai) déposition du seigneur Gaucerand, évêque de Trois-Châteaux et abbé de l'Île-Barbe. Que son âme repose en paix ».

III. Sous les décombres de l'abbatiale, on a trouvé au $\mathrm{XVII}^{\mathrm{e}}$ siècle la tombe de Fauque du Palais et Guillaume Ferlay :

\footnotetext{
HIC JACET FALQUA DE AULA UXOR QUONDAM GUILLELMI FERLAYS DOMICELLI FILII DOMINI... HIC JACET DOMINUS GUILLELMUS FERLAYS MILES QUONDAM QUI OBIIT MENSE AUGUSTI ANNO DOMINI MCCCIX
}

«Ci-gît Fauque du palais, femme de feu Guillaume Ferlay, damoiseau, fils du seigneur...

Ci-gît le seigneur Guillaume de Ferlay, jadis chevalier, qui mourut au mois d'août l'an du Seigneur 1309 ». 
IV. Dans le cloître, près de la grande porte de l'abbatiale se trouvait le monument de l'abbé Girin de Sartines (vers 12701299), et de Hugues de Ronchivol, Hugues de La Porte, Jean de Ronchivol, élevé par ce dernier en 1348 (n. st.) :

HIC JACET

DOMINUS GIRINUS DE SARTINES ABBAS INSULAE

ET HUGO DE RONCHIVOL PRIOR DE

POMMIERS

ET HUGO DE PORTA OBEDIENTIARUS DE

COLLUNGIIS

ET JOHANNES DE RONCHIVOL MONACHUS

QUI ELEGIT ISTAM SEPULTURAM ET FECIT PRO SE

ET SUIS

ACTUM MENSE MARTII ANNO DOMINI MCCCXLVII

ANIMAE EORUM REQUIESCANT IN PACE

«Ici gisent le seigneur Girin de Sartines, abbé de l'île, et Hugues de Ronchivol, prieur de Pommiers, et Hugues de La Porte, obédiencier de Collonges, et Jean de Ronchivol, moine, qui a choisi cette sépulture et l'a faite pour lui et les siens. Fait au mois de mars l'an du Seigneur 1347. Que leurs âmes reposent en paix ».

D'après le cartulaire de l'île-Barbe, Girin devient abbé vers 1270 , est encore cité comme tel le 25 avril 1299, est remplacé en septembre 1300. L'obéance de Collonges a été unie à l'office de cellérier en 1328, et le prieur de Collonges Hugues est dit à cette date défunt. En 1337 frère Jean de Ronchivol est cellérier de l'abbaye. 